\title{
Sexual behavior and fertility of male rats submitted to prolonged immobilization-induced stress
}

S.A. Almeida ${ }^{1}$

W.G. Kempinas ${ }^{2}$ and T.L. Lamano Carvalho ${ }^{3}$

\section{Correspondence}

T.L. Lamano Carvalho

Faculdade de O dontologia de

Ribeirão Preto, USP

Av. do Café $s / n$

14040-904 Ribeirão Preto, SP

Brasil

Fax: + 55-16-633-0999

E-mail: tllc@ forp.usp.br

Research supported by FAPESP

(No. 97/2498-1) and CNPq

(Nos. 520128/96-6 and 141513/95-0).

Received September 24, 1999

Accepted June 12, 2000

\author{
${ }^{1}$ Departamento de Fisiologia, Faculdade de Medicina de Ribeirão Preto, \\ Universidade de São Paulo, Ribeirão Preto, SP, Brasil \\ ${ }^{2}$ Departamento de Morfologia, Instituto de Biociências, \\ Universidade Estadual de São Paulo, Botucatu, SP, Brasil \\ ${ }^{3}$ Departamento de Morfologia, Estomatologia e Fisiologia, \\ Faculdade de $O$ dontologia de Ribeirão Preto, Universidade de São Paulo, \\ Ribeirão Preto, SP, Brasil
}

\section{Abstract}

In order to investigate whether prolonged stress interferes with the onset of sexual behavior at puberty and with fertility at adulthood, prepubertal male Wistar rats (40 days of age) were immobilized $6 \mathrm{~h}$ a day for 15 days (up to early puberty) or for 60 days (until sexual maturity). Pubertal stressed rats showed a two-fold increase in the latency for the first mount (probably due to repeated aversive experience in which a change of environment was always followed by immobilization) and a 2.5 -fold increase in the frequency of thrusting (indicative of enhanced sexual performance). The apparently stimulatory effect of prolonged stress on the onset of sexual behavior is discussed in terms of increased testosterone level and interference with the complex interchanges between the neurotransmitters/neuropeptides involved in the central control of male sexual activity. Adult stressed animals were mated with normal females, which became pregnant but exhibited a more than two-fold increase in both preimplantation and post-implantation loss, probably due to a smaller rate of fertilization and/or fertilization with damaged spermatozoa.

\section{Introduction}

As a result of hypothalamic-pituitary-adrenal axis activation, prolonged stress may inhibit the male reproductive functions through a depression of the hypothalamicpituitary-testicular axis (1). Previous studies from our laboratory have shown that chronic intermittent immobilization-induced stress applied to male rats from prepuberty caused

\section{Key words} - Immobilization stress

- Sexual behavior

- Male fertility a significant decrease in plasma LH of both pubertal and adult rats, whereas plasma testosterone was lower than control levels in adult stressed rats but was more than twofold higher in pubertal animals (2-4), suggesting that prolonged stress probably acts in a different way on the gonadal axis during distinct phases of sexual development.

Since adrenergic innervation seems to play a pivotal role in testicular steroidogen- 
esis around the onset of puberty (5), we proposed that sympathetic overstimulation might explain the increased testosterone levels observed in pubertal stressed rats. Prolonged immobilization caused no significant change in plasma FSH but induced a significant delay in testicular maturation, in addition to a decrease in spermatid production and in sperm density in both pubertal and adult animals $(3,4)$. Few literature data pertaining to stress and sexual behavior have shown that prenatal $(6)$ or neonatal $(7,8)$ stimulation may impair the copulatory performance of both male and female adult rats. The purpose of the present study was to determine whether prolonged immobilization-induced stress applied from prepuberty interferes with the onset of sexual behavior at puberty and with fertility during adulthood.

\section{Material and Methods}

Prepubertal (40 days of age) male Wistar rats were housed under controlled environmental conditions (12-h light period and temperature of $23 \pm 2^{\circ} \mathrm{C}$ ) and had free access to laboratory chow and tap water. Stress was attained by immobilization inside plastic tubes for $6 \mathrm{~h}$ a day during the light period, starting at 8:00 a.m., for 15 days (early pubertal animals used for analysis of sexual behavior) or for 60 days (sexually mature rats used in fertility experiments) (see Ref. 9, for the phases of male rat sexual development). Control animals were left undisturbed in their cages.

\section{Sexual behavior}

For analysis of the onset of sexual behavior, pubertal control and stressed males $(\mathrm{N}=$ 8 per group) with no previous sexual experience were placed with adult receptive females in natural estrus during a 30-min session. The males were placed in the observation cage $(56 \times 35 \times 31 \mathrm{~cm}$ with a glass front wall) $2 \mathrm{~h}$ after the beginning of the dark phase and $10 \mathrm{~min}$ before the females, for adaptation to the environment. The latency and quantity of mounts and thrusting were recorded simultaneously by two investigators, with light provided by a 40 -watt red lamp. In the mount behavior the male places his forepaws on the female without pelvic movements, while in the thrusting behavior he executes repeated deep pelvic thrusts (10).

\section{Fertility assessment}

Adult control and stressed males $(\mathrm{N}=10$ per group) were mated with sexually mature normal females presenting at least three regular cycles confirmed by analysis of daily vaginal smears. Females in the proestrus stage in the morning were mated with males overnight ( 2 females per male); the presence of spermatozoa in the vaginal smear on the next morning was indicative of copulation and was considered as day zero of pregnancy. Maternal autopsy was performed on day 20 of pregnancy when uterus and ovaries were examined for number of corpora lutea, dead and live fetuses, embryo resorption and implantation sites (total amount of live and dead fetuses plus embryo resorptions) (see Ref. 11, for methodological details). The rates of pre-implantation loss (corpora lutea minus implantation sites) and postimplantation loss (implantation sites minus live fetuses) were then determined.

\section{Results}

Compared to control animals, pubertal stressed rats exhibited an increased latency for the first mount followed by an increased frequency of thrusting (Figure 1). Neither control nor stressed animals ejaculated during this period.

All but one female mated with adult control or stressed males became pregnant. Data from maternal autopsy carried out on day 20 of pregnancy (Table 1) were used to estimate 
fertility rates, which showed that the females mated with stressed males exhibited a significant increase in both pre-implantation and post-implantation loss (Figure 2).

\section{Discussion}

A previous study was carried out to investigate the prolonged intermittent immobilization stress protocol applied throughout male rat sexual development, focusing on food intake, body weight gain, and stressrelated and sex hormones (2). The results confirmed the presence of a sustained stress condition on the basis of a significant rise in plasma ACTH and corticosterone following both 15 and 60 days of immobilization. When applied from prepuberty (40 days of age) to early puberty ( 55 days of age) the stressful stimuli significantly increased plasma testosterone but delayed testicular maturation (4). When applied from 40 to 100 days of age (full sexual maturity) immobilization stress caused a marked drop in plasma testosterone besides a reduction in the amount of mature spermatids in the testis and in spermatozoon concentration in the cauda epididymidis (3). The present experiment was conducted to investigate whether prolonged immobilization stress interferes with the onset of sexual behavior at puberty and with fertility at adulthood.

Compared to controls, pubertal stressed rats exhibited an increased latency for the first mount that might be suggestive of a lower sexual motivation (12) but may also be related to a repeated aversive experience in which a change of environment was always followed by immobilization. Stressed animals presented also an increased frequency of thrusting, indicative of enhanced sexual performance since neither control nor stressed animals ejaculated.

Since the time immediately after birth is critical for brain development, numerous studies have examined the long-lasting effects of neonatal stimulation on many emo-

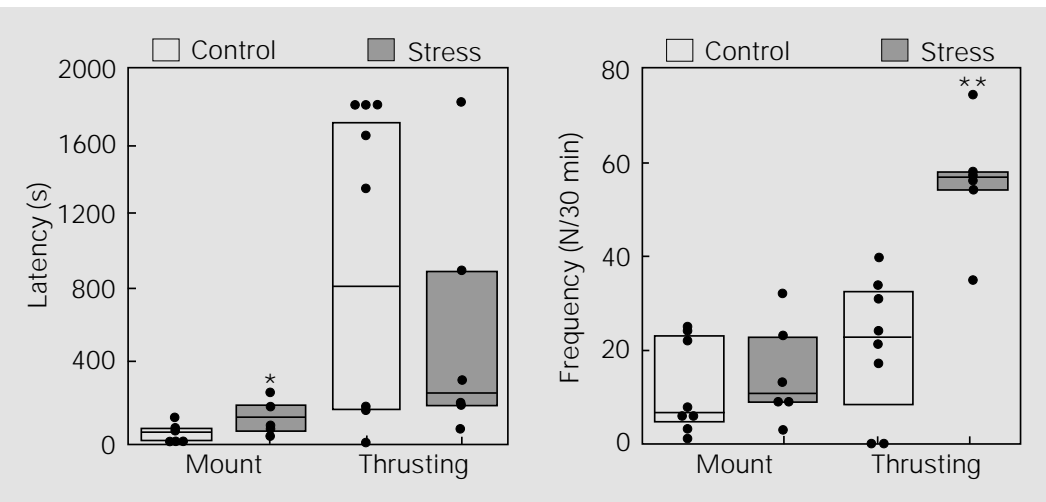

Figure 1 - Latency and frequency of mounts and thrusting in control and stressed pubertal rats with no previous sexual experience placed with adult receptive females during a 30min session. Stress was attained by immobilization for $6 \mathrm{~h}$ a day from prepuberty (40 days of age) to early puberty ( 55 days of age). Upper and lower boundaries mark the 25 and $75 \%$ percentiles and the intermediate horizontal bars are the median values. $* \mathrm{P}=0.01, * * \mathrm{P}=$ 0.05 compared to the control group (Mann-Whitney test).

Table 1 - Total amount of corpora lutea, live and dead fetuses, and resorption and implantation sites observed in pregnant females mated with control and stressed males.

\begin{tabular}{lcccccc}
\hline Males & \multicolumn{6}{c}{ Total amount } \\
\cline { 2 - 7 } & $\begin{array}{c}\text { Pregnant } \\
\text { females }\end{array}$ & $\begin{array}{c}\text { Corpora } \\
\text { lutea }\end{array}$ & $\begin{array}{c}\text { Live } \\
\text { fetuses }\end{array}$ & $\begin{array}{c}\text { Dead } \\
\text { fetuses }\end{array}$ & $\begin{array}{c}\text { Resorption } \\
\text { sites }\end{array}$ & $\begin{array}{c}\text { Implantation } \\
\text { sites }\end{array}$ \\
\hline Control $(\mathrm{N}=10)$ & 19 & 275 & 247 & 0 & 15 & 262 \\
Stress $(\mathrm{N}=10)$ & 20 & 293 & 218 & 13 & 23 & 254
\end{tabular}

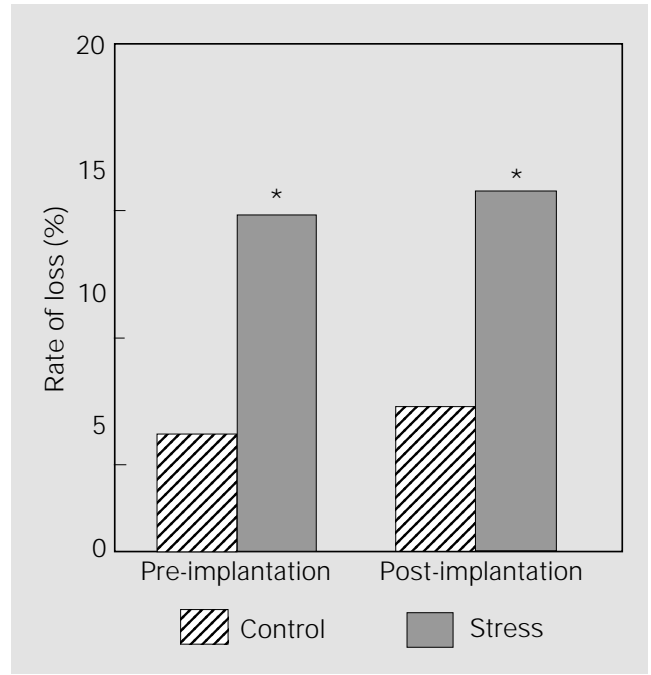

Figure 2 - Rates of pre-implantation and post-implantation loss in normal females mated with control and stressed adult rats. Stress was attained by immobilization for $6 \mathrm{~h}$ a day from prepuberty (40 days of age) to full sexual maturity (100 days of age). The rates of pre-implantation loss (corpora lutea minus implantation sites) and post-implantation loss (implantation sites minus live fetuses) were determined on day 20 of pregnancy. $* \mathrm{P}=0.01$ compared to the control group (chi-square test). 
tional responses and behaviors in adulthood. In contrast to our results, literature data have shown a decreased sexual behavior in both male and female prepubertal and adult rats submitted to handling or more aversive stimuli during early postnatal life $(7,8)$.

Many central neurotransmitters and neuropeptides are involved in the control of male sexual behavior. Increased brain noradrenergic and dopaminergic activities may improve parameters of copulatory activity, indicating their facilitatory role in the process $(12,13)$. Although early studies have proposed an opposite role for serotonin (13), more recent data have shown that 5-HT1A and 5-HT2 receptor agonists may also stimulate sexual motivation and completion (10,1416). The effects of serotonin and dopamine on male copulatory behavior seem to occur by interaction with testosterone $(10,12,14$, 16). A proper androgenic status is also necessary for a normal sexual performance, the deleterious effects of castration being reversed by hormonal replacement; moreover, chronic treatment of prepubertal rats with testosterone can precipitate the onset of first mount, thrusting and ejaculation, probably by stimulation of sexual arousal (13). It is possible that increased plasma testosterone concentration $(2,4)$, in addition to the higher catecholamine and serotonin levels expected to occur after prolonged stress (1), might account for the enhanced sexual performance herein described at the onset of puberty.

Adult animals submitted to intermittent immobilization from prepuberty presented a significant decrease in fertility rates, confirmed by a more than 2 -fold increase in both pre-implantation and post-implantation loss in the progeny of normal females. Pre-implantation loss corresponds to the sum of unfertilized ova and/or embryo loss occurring before endometrial implantation, whereas post-implantation loss refers to a later impairment of embryo-fetal viability. The reduced fertility of stressed males is probably due to a lower rate of ovum fertili- zation and/or fertilization with damaged spermatozoa.

There are some reports about the deleterious effects of stress on animal and human semen quality, such as a reduction in seminal volume, in sperm concentration and motility, and in the amount of normal spermatozoa (17-20). These manifestations are less obvious in men, being generally associated with extreme physical or psychological situations and considered to be the basis of temporary infertility (19). Although an analysis of spermatozoon morphology and motility was not performed in the present study, the possibility of altered parameters cannot be ruled out.

It is known that the production of spermatozoa able to fertilize and to develop a normal progeny results, at least in part, from normal sperm maturation in the epididymis. The composition of the internal epididymal milieu, responsible for sperm maturation, is under androgen control. In rats, an androgen-binding protein secreted by Sertoli cells into the lumen of seminiferous tubules under FSH stimulation is transported to the epididymis, where it accumulates at concentrations higher than those found in the testes. This leads to a high local concentration of androgens, essential for maturation of epididymal spermatozoa (17).

We have previously reported that adult male rats exposed to prolonged immobilization exhibit a decrease in spermatid production and sperm density, in addition to lower plasma testosterone concentration (3) and a small but significant reduction in the amount of Sertoli cells per seminiferous tubule crosssection (from $14.1 \pm 0.2$ in controls to $12.5 \pm$ 0.3 in stressed animals; $\mathrm{P}=0.01$, MannWhitney test). Thus, the possibility of a disturbance in the maturation of spermatozoa should be considered. If so, the reduced sperm density, side by side with fertilization by immature and/or damaged spermatozoa, might explain the reduced fertility observed in stressed males. 
In conclusion, prolonged intermittent immobilization stress applied to male rats from prepuberty enhances sexual performance at the onset of puberty but decreases fertility at sexual maturity.

\section{References}

1. Rivier C \& Rivest S (1991). Effect of stress on the activity of the hypothalamic-pituitary-gonadal axis: peripheral and central mechanism. Biology of Reproduction, 45: 523-532.

2. Almeida AS, Anselmo-Franci J A, Rosa-eSilva AAM \& Lamano Carvalho TL (1998). Chronic intermittent immobilization of male rats throughout sexual development: a stress protocol. Experimental Physiology, 83: 701-704.

3. Almeida AS, Petenusci SO, AnselmoFranci J A, Rosa-e-Silva AAM \& LamanoCarvalho TL (1998). Decreased spermatogenic and androgenic testicular functions in adult rats submitted to immobilizationinduced stress from prepuberty. Brazilian J ournal of Medical and Biological Research, 31: 1443-1448.

4. Almeida AS, Petenusci SO, AnselmoFranci J A, Rosa e Silva AAM \& Lamano Carvalho TL (2000). Chronic immobilization-induced stress increases plasma testosterone and delays testicular maturation in pubertal rats. Andrologia, 32: 7-11.

5. Lamano Carvalho $T L$, Guimarães $M A$, Kempinas WG, Petenusci SO \& Rosa e Silva AAM (1996). Effects of guanethidine-induced sympathectomy on the spermatogenic and steroidogenic testicular functions of prepubertal to mature rats. Andrologia, 28: 117-122.

6. Ward IL, Ward OB, Winn RJ \& Bielawski D (1994). Male and female sexual behavior potential of male rats prenatally ex-

\section{Acknowledgments}

The authors thank A. Paris, F.B. Sarro, R. Mazaro and G.S. Romualdo for help with the analysis of sexual behavior and fertility. posed to the influence of alcohol, stress or both factors. Behavioral Neuroscience, 108: 1188-1195.

7. Lucion $A B$, Cadore LP, Charchat $H$, Barros HMT \& Padoin MJ (1997). Effects of noxious stimulation during the stress-hyporesponsive period on behaviors of pre-pubertal and adult male and female rats. Proceedings of the 27th Annual Meeting of the Society for Neurosciences, New Orleans, LA, USA, 1081.

8. Frantz PJ, Gomes CM, Sanvitto GL \& Lucion AB (1999). Efeito da estimulação neonatal sobre o comportamento sexual de ratas. Proceedings of the XIV Reunião Anual da Federação das Sociedades de Biologia Experimental, August 25-28, Caxambu, MG, Brazil, Abstract 01.396.

9. Zanato VF, Martins MP, Anselmo-Franci J A, Petenusci SO \& Lamano-Canvalho TL (1994). Sexual development of male Wistar rats. Brazilian J ournal of Medical and Biological Research, 27: 1273-1280.

10. Padoin MJ \& Lucion AB (1995). The effect of testosterone and $\mathrm{DOI}$ on male sexual behavior of rats. European J ournal of Pharmacology, 277: 1-6.

11. Taylor P (1986). Maternal necropsy and foetal examination. In: Practical Teratology. Academic Press, New York.

12. Melis MR \& Argiolas A (1995). Dopamine and sexual behavior. Biobehavioral Reviews, 19: 19-38.

13. Meisel RL \& Sachs BD (1994). The physiology of male sexual behavior. In: Knobil E
\& Neil J D (Editors), The Physiology of Reproduction. Raven Press, London.

14. Rasia-Filho AA \& Lucion AB (1996). Effects of 8-OH-DPAT on sexual behavior of male castrated at different ages. Hormones and Behavior, 30: 251-258.

15. Femandez-Guasti $A \&$ Rodriguez-Manzo G (1997). 8-OH-DPAT and male rat sexual behavior: partial blockade by noradrenergic lesion and sexual exhaustion. Pharmacology, Biochemistry and Behavior, 56: 111-116.

16. Rowland DL \& Houtsmuller EJ (1998). 8OH-DPAT interacts with sexual experience and testosterone to affect ejaculatory response in rats. Pharmacology, Biochemistry and Behavior, 60: 143-149.

17. Steinberger E (1978). The etiology and pathophysiology of testicular dysfunction in man. Fertility and Sterility, 29: 481-491.

18. Giblin PT, Poland ML, Moghissi KS, Ager J W \& Olson J M (1988). Effects of stress and characteristic adaptability on semen quality in healthy men. Fertility and Sterility, 49: 127-132.

19. Negro-Vilar A (1993). Stress and other environmental factors affecting fertility in men and women: overview. Environmental and Health Perspectives, 101 (Suppl 2): 59-64.

20. Cui KH (1996). The effect of stress on semen reduction in the marmoset monkey (Callithrix jacchus). Human Reproduction, 11: 568-573. 